\title{
Defect Spectroscopy in Halide Perovskites Is Dominated by Ionic Rather than Electronic Defects
}

Cite This: ACS Energy Lett. 2022, 7, 140-144

Read Online

Article Recommendations

A tomic defects in semiconductors such as vacancies, interstitials, or dopants can lead to additional electronic states within the bandgap. These atomic defects are considered immobile in classical semiconductor theory, one of the reasons why a $\mathrm{p}-\mathrm{n}$ junction with its ionized dopants remains stable over time. For mixed ionic-electronic conductors, the assumption of immobile defects is not valid. Halide perovskites ( $\mathrm{HaPs}$ ) are a class of materials in which ions and the related atomic defects have been shown to be mobile and migrate in response to external stimuli such as voltage or light. ${ }^{1-3}$ We refer to them as ionic defects. Using iodide partial pressure investigations, it was deduced that the halide ion is the most mobile. ${ }^{4,5}$ Furthermore, it was shown that the mobile ions follow the Meyer-Neldel rule ${ }^{6,7}$ and that HaPs are capable of self-healing after being damaged. ${ }^{8,9}$ As a result, Rakita et al. ${ }^{10}$ stated that HaPs "are likely an example of a class of materials that cannot support static bulk defect densities significantly above thermodynamically-dictated densities. The reasons are (a) the free energy to form HaPs (from binary halides) is less than the formation energies of (static) defects in them and (b) the small kinetic stabilization of such defects." These dynamic defects have significant implications for defect spectroscopy measurements. ${ }^{11,12}$ In defect spectroscopy, the observed electronic defects (in the bandgap) that trap (localize) mobile charge carriers are usually assumed to be caused by immobile ionic defects. In the case of mobile ionic defects, it is instead possible to observe the migration of the ionic defects themselves, rather than the capture/emission of charge carriers into/from the electronic defects created by these ionic defects.

In this Viewpoint, we discuss the difference between ionic and electronic defects, how to distinguish between them with transient techniques, and the implications of the mixed ionicelectronic conduction on the most commonly used (opto)electronic measurement techniques.

Electronic Vs Ionic Defects. Electronic and ionic defects often lead to similar experimental observable signals. To understand how to distinguish between them, one must consider the time scales involved in the different processes (Figure 1). In electronic defects (which are created by static or, indeed, mobile ionic defects), electrons are captured at point defects or extended defects acting as a trap. According to

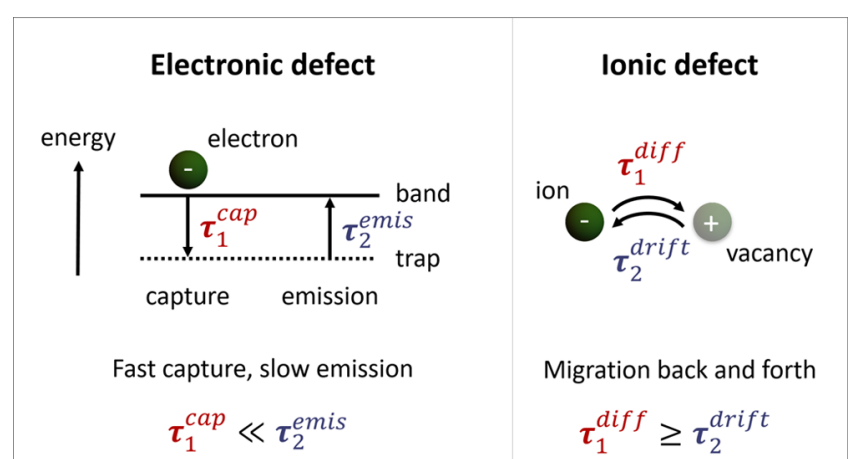

Figure 1. Difference in time scales between electronic and ionic defects arises from their different physical origins. For electronic defects, it is the capture and emission of electrons (or holes) at electronic trap states within the bandgap. For ionic defects, it is the migration of mobile ions back and forth.

Shockley-Read-Hall theory, ${ }^{13,14}$ the capture rate for electrons $1 / \tau_{1}^{\text {cap }}$ is defined as

$$
\frac{1}{\tau_{1}^{\text {cap }}}=\sigma_{\mathrm{n}} \nu_{\mathrm{th}} N_{\mathrm{C}}
$$

where $\sigma_{\mathrm{n}}$ is the capture cross section for capturing electrons, $\nu_{\text {th }}$ the thermal velocity, and $N_{\mathrm{C}}$ the density of states in the conduction band. Typical capture times are between $10^{-13}$ and $10^{-10} \mathrm{~s}$ at room temperature. The subsequent temperaturedependent emission rate, $1 / \tau_{2}^{\text {emis }}$, of the captured electrons is defined as

$$
\frac{1}{\tau_{2}^{\text {emis }}}=\frac{1}{\tau_{1}^{\text {cap }}} \exp \left(-\frac{E_{\mathrm{T}}}{k_{\mathrm{B}} T}\right)
$$

where $E_{\mathrm{T}}$ is the energy difference between the conduction band and trap level, $k_{\mathrm{B}}$ Boltzmann's constant, and $T$ the temperature. We note that $E_{\mathrm{T}}$ is often also referred to as

Received: September 24, 2021

Accepted: November 19, 2021 
activation energy. An analogous expression holds for the capture and emission of holes. The emission time therefore depends strongly on the activation energy, with expected times between $10^{-10} \mathrm{~s}$ for shallow traps and $10^{0} \mathrm{~s}$ at room temperature for deep traps with activation energies up to 0.6 $\mathrm{eV} .{ }^{12}$

For ionic defects, the time scales are determined by the migration of mobile ions facilitated by ionic defects such as vacancies or interstitials. Charged species such as mobile ions can migrate by thermally activated processes in response to concentration gradients (diffusion) and electric fields ( drift $^{15}$ ). One can identify two extreme cases: One where migration is governed only by drift and one where migration is governed only by diffusion. ${ }^{16}$ For diffusion, the time scale is given by

$$
\frac{1}{\tau_{1}^{\text {diff }}}=\frac{D}{d^{2}}=\frac{D_{0}}{d^{2}} \exp \left(-\frac{E_{\mathrm{A}}}{k_{\mathrm{B}} T}\right)
$$

where $D$ is the average diffusion coefficient of mobile ions, $d$ their diffusion length, $D_{0}$ a temperature-independent parameter, and $E_{\mathrm{A}}$ the activation energy for diffusion. ${ }^{17}$ For mobile ionic defects, the activation energy for the migration rate is the same as for average ion diffusion: It is essentially the sum of the ionic defect (pair) formation and migration enthalpies. For drift, the time scale can be expressed as

$$
\frac{1}{\tau_{2}^{\text {drift }}}=\frac{\mu F}{d}=\frac{1}{\tau_{1}^{\text {diff }}} \frac{V}{k_{\mathrm{B}} T}
$$

where $\mu$ is the mobility of mobile ions and $F=V / d$ the electric field across the layer of interest. We note that $d$ here is assumed to be the thickness of this layer; i.e., the diffusion length is limited by the layer thickness. To write $\tau_{2}^{\text {drift }}$ as a function of $\tau_{1}^{\text {diff }}$, the Einstein relation $D / \mu=k_{\mathrm{B}} T / e$ is used. The expected time scales for drift and diffusion are on the order of milliseconds to seconds, with drift being somewhat faster depending on the applied field.

From eqs $1-4$, one can see a clear difference between time scales for electronic and ionic defects. For electronic defects, the ratio between emission and capture rate is much smaller than 1, i.e., $\tau_{1}^{\text {cap }} / \tau_{2}^{\text {emis }} \ll 1$. The difference between $\tau_{1}^{\text {cap }}$ and $\tau_{2}^{\text {emis }}$ is exponentially dependent on both the temperature and the energetic position of the trap. For ionic defects, on the other hand, $\tau_{1}^{\text {diff }} / \tau_{2}^{\text {drift }} \geq 1$ applies. The maximum difference between $\tau_{1}^{\text {diff }}$ and $\tau_{2}^{\text {drift }}$ is only linearly dependent on the bias and the temperature; for small electric fields, they may approximately be the same. As results, the difference between $\tau_{1}$ and $\tau_{2}$ is much higher for electronic defects than it is for ionic defects. Hence, measuring both $\tau_{1}$ and $\tau_{2}$ allows for a distinction between electronic and ionic defects to be made.

The most established methods for measuring electronic defects in semiconductors are deep-level transient spectroscopy (DLTS) and impedance spectroscopy (IS). ${ }^{18,19}$ In both DLTS and IS a capacitance response is measured after applying a voltage perturbation either in the time or in the frequency domain. In a DLTS measurement, the capacitance transient after a forward voltage pulse $V_{\mathrm{b}}-V_{\mathrm{a}}>0 \mathrm{~V}$ ends, i.e., after the step from $V_{\mathrm{b}}$ back to $V_{\mathrm{a}}$, yields the time constant $\tau_{2}$ (see Figure 2 ). Reverse DLTS (R-DLTS) measures the inverse process with the time constant $\tau_{1}$ of the capacitance transient following the voltage step from $V_{\mathrm{a}}$ to $V_{\mathrm{b}}$. We note that (i) for electronic defects, the forward pulse to $V_{\mathrm{b}}$ corresponds to trap filling (capture), so that after the pulse the emission from these electronic defects is observed; (ii) for ionic defects, $V_{\mathrm{b}}$ is

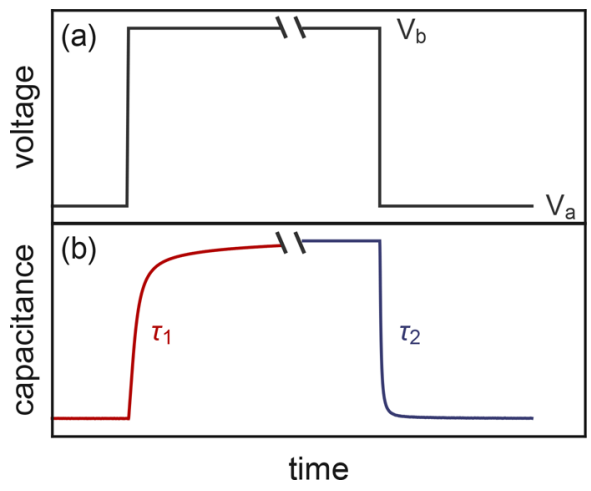

Figure 2. Schematic illustration of (a) the applied voltage and (b) the obtained capacitance response in a DLTS/TID measurement to distinguish between ionic and electronic defects. The time scales $\tau_{1}$ and $\tau_{2}$ can be obtained by fitting the measured capacitance response with exponential decay functions or by an inverse Laplace transform.

usually chosen to cancel out the internal electric field (flatband conditions), hence $\tau_{1}$ corresponds to the migration of mobile ions without a field, i.e., diffusion, and $\tau_{2}$ to the migration within the internal electric field by drift; and (iii) for ionic defects, the bias needs to be applied long enough (typically in the order of seconds) to allow the ions to fully redistribute during the measurement. Figure 3 shows measured

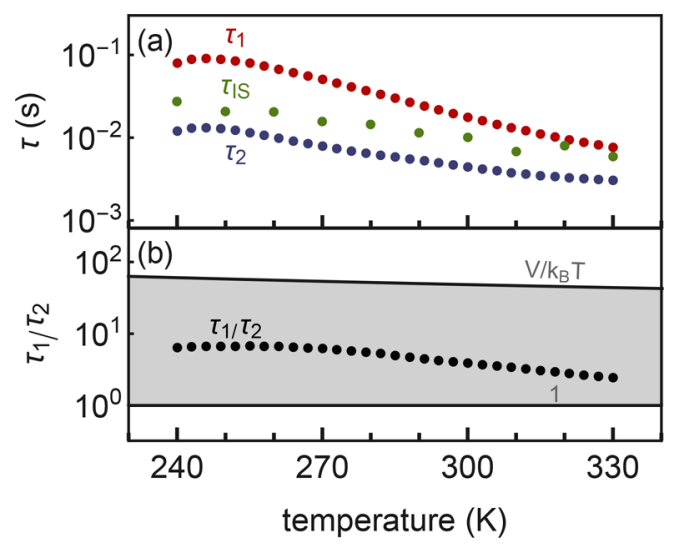

Figure 3. (a) Determined time scales of a halide perovskite material measured in the time domain with DLTS/TID and in the frequency domain with IS. (b) The ratio of $\tau_{1} / \tau_{2}$ is between 1 and $V / k_{\mathrm{B}} T$, confirming the ionic nature of the measured signals for both DLTS and IS. Data from ref 12 .

time scales with DLTS and IS of a HaP material $\left(V_{\mathrm{a}}=0 \mathrm{~V}\right.$ and $\left.V_{\mathrm{b}}=1.25 \mathrm{~V}\right)$. The ratio $\tau_{1} / \tau_{2}$ obtained in the time domain is above 1 , confirming that the measured process is related to mobile ion migration and not to electronic defects. This assignment is only possible in the time domain, where both $\tau_{1}$ and $\tau_{2}$ can be measured independently. In the frequency domain, usually only the slower process or a superposition of several processes is visible, which makes the distinction impossible.

Implications. The most commonly used (opto)electronic methods to investigate charge carrier dynamics in semiconducting materials are summarized in Table 1. All these methods are used to study various processes such as emission, trapping, transport, or recombination in semiconductors without taking mobile defects into account. However, in the 
Table 1. Most Commonly Used (Opto)electronic Methods to Investigate Defects and Charge Carrier Dynamics in Semiconducting Materials ${ }^{a}$

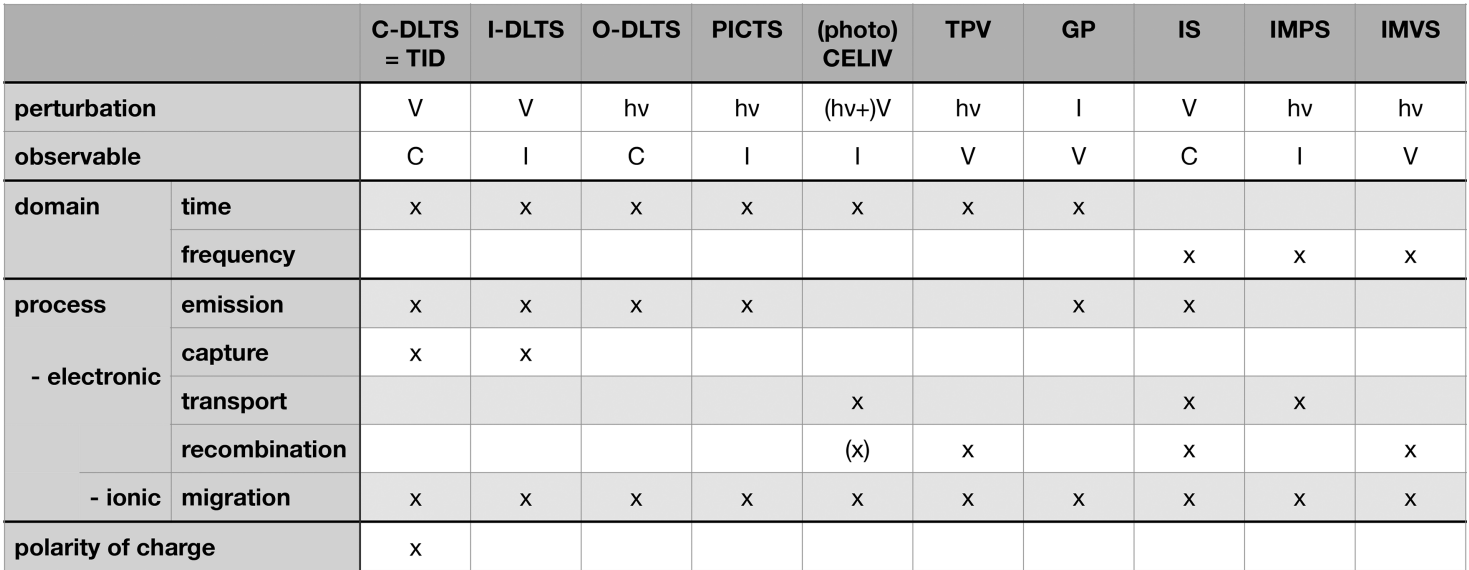

${ }^{a}$ The electronic processes which are typically observed with these methods are highlighted. We anticipate that all of the methods show contributions due to ion migration. The perturbation is by voltage $V$, light $h \nu$, or current $I$. The observable is capacitance $C$, current, or voltage. Only the measurement of the capacitance transient allows to distinguish between the polarities of the charge. PICTS is comparable to (biasassisted) charge extraction and transient photocurrent. Abbreviations: CELIV, charge extraction by linearly increasing voltage; TPV, transient photovoltage; GP, galvanostatic polarization; IMPS and IMVS, intensity modulated photocurrent/photovoltage spectroscopy. IS and thermal admittance spectroscopy are equivalent.

case of mixed ionic-electronic conductors, these methods also yield possible signals originating from the migration of ionic defects, the density of which is ranging from $10^{12}$ to $10^{19}$ $\mathrm{cm}^{-3}$. For the frequency domain, the classical theory of thermal admittance spectroscopy from Walter et al. ${ }^{18}$ to explain IS measurements in terms of electronic defect states cannot be applied to ionic defects. Instead, the capacitance increase at low frequencies is mainly related to ion migration and polarization of the ion-blocking electrode-an effect wellknown in ionic conductors. ${ }^{20,21}$ This redistribution of mobile ions was furthermore shown to modulate electronic currents. $^{22-24}$ Also for the time domain, it was shown that the classical DLTS theory is in many cases not valid for mixed conductors as the measured signals are often a combination of both electronic and ionic defects. ${ }^{11,12}$ We note that the timedomain methods DLTS and transient ion drift (TID) ${ }^{16}$ are experimentally identical, but classical DLTS theory interprets all results as electronic defects, while TID interprets them as ionic defects. Both DLTS and TID are hence often used interchangeably in literature when measuring ionic defects, and have been successfully used to identify and quantify ionic defects in $\mathrm{Cu}$-doped $\mathrm{Si}^{16}{ }^{16} \mathrm{CdTe}$ and $\mathrm{CuInSe}{ }_{2},{ }^{25}$ and $\mathrm{HaPs}^{26,27}$ We point out that several researchers have done important work on understanding ionic defects in halide perovskites. ${ }^{1,3-5}$ Nevertheless, it is generally the task of the experimentalist to ensure that the measurement signal from mixed conductors is either electronic or ionic in nature.

As an example, we consider the ionic defect $\beta$, which has been reported by different researchers ${ }^{6,26,28-31}$ for various $\mathrm{HaPs}$ and has been attributed to both an ionic and electronic defect (see Figure 4). We confirmed that the migration rates for capture/diffusion (R-DLTS) and emission/drift (DLTS) are very similar, $\tau_{1} \approx \tau_{2}$, not only for $\beta$ but for all defects we observed. $^{27}$ This finding shows that ionic defects are observed rather than electronic ones (for which capture rates are much higher than emission rates with the observed activation energies). Figure 4 (bottom) illustrates that different experimental methods with various perturbations (here,

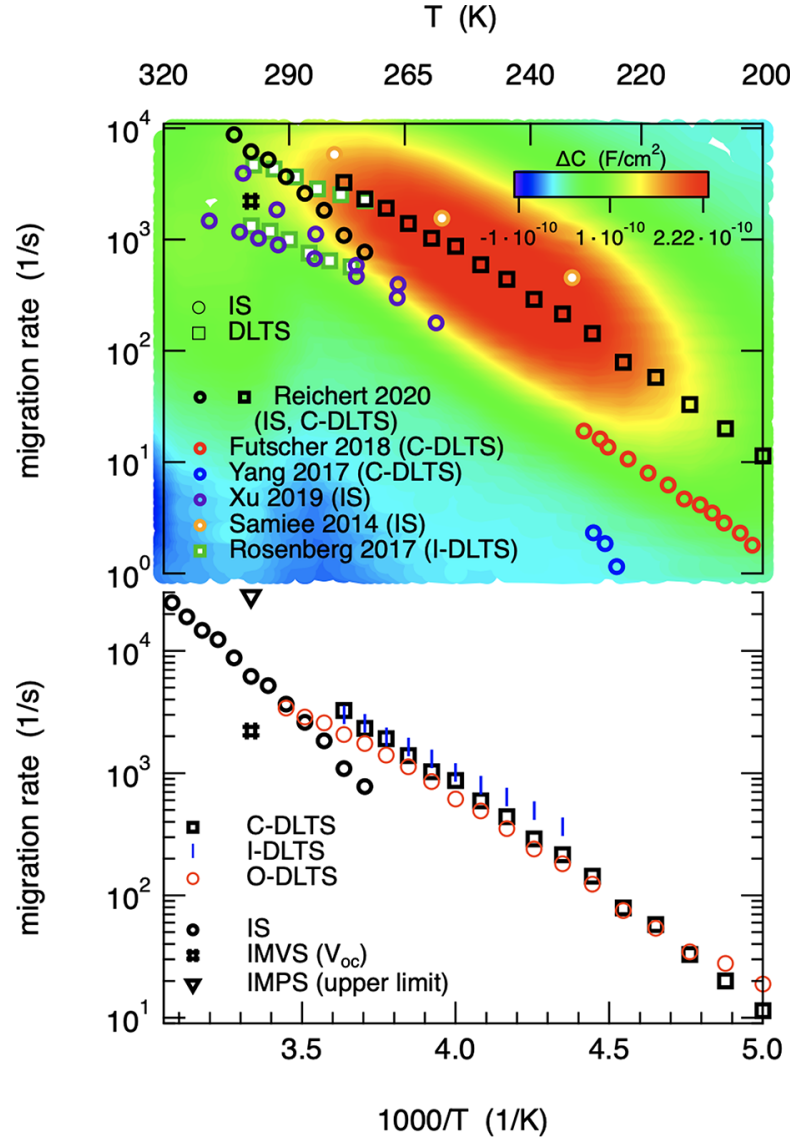

Figure 4. Arrhenius plots for migration rates of the ionic defect $\beta$. (Top) Migration rates for metal halide perovskites from literature in the range where we measure $\beta \cdot{ }^{6,26,28-31}$ The contour plot in the background shows DLTS boxcar spectra. ${ }^{27}$ (Bottom) Comparison of selected time domain and frequency domain methods. Data from refs 27 and 32. 
voltage or light) and observables (here, capacitance, current, or photovoltage/photocurrent) in both the frequency and time domain yield comparable signals from this ionic defect $\beta$. This finding strongly suggests that the ionic response masks electronic defects in $\mathrm{HaPs}$, whether voltage or light pulses cause the disturbance. Great care must therefore be taken to verify whether the measured signals are due to electronic or ionic defects, as this may otherwise lead to incorrect conclusions.

Indeed, the similarity of ionic and electronic defects has led to numerous controversies in literature. For example, IS or DLTS have frequently been used to study electronic defects in HaPs. ${ }^{29,33-36}$ Yet, as mentioned above, the measured signals are likely to be due to ionic rather than electronic defects, with important consequences. For example, Pecunia et al. recently reported that photoinduced current transient spectroscopy (PICTS) "offers a facile, widely applicable route to the defectlevel characterization", referring to electronic defects. ${ }^{37}$ Because the HaPs considered are known ion conductors, we believe it essential to confirm that emission rate $\ll$ capture rate, to propose that indeed electronic defects are observed. If this is not done, it is likely that ionic defects are being measured, and not the electronic processes related to these ionic defects.

Similar to HaPs, solar cells based on the semiconductor $\mathrm{CuInSe}_{2}$ (CIS in short; and its variants with $\mathrm{Ga}$ and $\mathrm{S}$ ) are known to contain mobile ionic defects. ${ }^{25}$ For instance, Rau et al. $^{38}$ stated that "Cu is well known to be a mobile constituent in CIS", which was confirmed also by the radioactive tracer technique. $^{39}$ Still, the assignment of at least one defectspectroscopic signal in CIS has remained under debate: The defect N1, observed by IS and DLTS independent of the choice of transport layers. ${ }^{40,41}$ It shows varying transition energies ${ }^{42}$ depending on bias, ${ }^{43}$ air annealing or damp-heat treatment, and cannot be a classical monoenergetic electronic defect as the rates for emission/drift (DLTS) and capture/ diffusion (R-DLTS) have been reported to be equal. ${ }^{44}$ Similar to $\beta$ in metal halide perovskites, ${ }^{6}$ the $\mathrm{N} 1$ defect in CIS follows the Meyer-Neldel rule. ${ }^{45}$ Therefore, N1 has been interpreted as continuum of donor states at the CIS surface ${ }^{42}$ or an injection/extraction barrier. ${ }^{46,47}$ In view of the evidence for $\mathrm{Cu}$ migration in CIS, N1 showing up with same rates for $\tau_{1}$ and $\tau_{2}$ independent of any transport layers, and ionic defects often following the Meyer-Neldel rule, ${ }^{7}$ we propose that the N1 signal in CIS corresponds to ionic migration, likely to $\mathrm{Cu}$ related defects.

\section{Take-Away Messages.}

- In mixed ionic-electronic conductors such as HaPs or CISs, the ionic responses often mask electronic defects, regardless of whether a voltage pulse, a current pulse, or a light pulse causes the disturbance, or whether current, capacitance, or voltage is the quantity to be observed.

- When performing opto(electronic) measurements on mixed conductors, one must make sure whether the measured signals are due to ionic or electronic defects, as otherwise erroneous conclusions may be drawn.

- Only if it can be ruled out that the signal is due to ionic defects can electronic defect characteristics be determined.

Moritz H. Futscher 우 orcid.org/0000-0001-8451-5009

Carsten Deibel $\odot$ orcid.org/0000-0002-3061-7234

\section{AUTHOR INFORMATION}

Complete contact information is available at: https://pubs.acs.org/10.1021/acsenergylett.1c02076

\section{Notes}

Views expressed in this Viewpoint are those of the authors and not necessarily the views of the ACS.

The authors declare no competing financial interest.

\section{ACKNOWLEDGMENTS}

The authors would like to thank David Cahen, Romain Carron, and Alessandro Senocrate for carefully reading and commenting on the manuscript. M.H.F. is supported by a Rubicon Fellowship from the Dutch Research Council (NWO). C.D. thanks the German Research Foundation (DFG) for support within the framework of the SPP 2196 project PERFECT PVs.

\section{REFERENCES}

(1) Walsh, A.; Stranks, S. D. Taking control of ion transport in halide perovskite solar cells. ACS Energy Letters 2018, 3, 1983-1990.

(2) Cohen, A. V.; Egger, D. A.; Rappe, A. M.; Kronik, L. Breakdown of the static picture of defect energetics in halide perovskites: The case of the $\mathrm{Br}$ vacancy in $\mathrm{CsPbBr}_{3}$. J. Phys. Chem. Lett. 2019, 10, 4490-4498.

(3) Futscher, M. H.; Milić, J. V. Mixed conductivity of hybrid halide perovskites: Emerging opportunities and challenges. Front. Energy Res. 2021, 9, 629074.

(4) Senocrate, A.; Maier, J. Solid-state ionics of hybrid halide perovskites. J. Am. Chem. Soc. 2019, 141, 8382-8396.

(5) Leupold, N.; Seibel, A. L.; Moos, R.; Panzer, F. Electrical conductivity of halide perovskites follows expectations from classical defect chemistry. Eur. J. Inorg. Chem. 2021, 2021, 2882-2889.

(6) Reichert, S.; An, Q.; Woo, Y.-W.; Walsh, A.; Vaynzof, Y.; Deibel, C. Probing the ionic defect landscape in halide perovskite solar cells. Nat. Commun. 2020, 11, 6098.

(7) Gelin, S.; Champagne-Ruel, A.; Mousseau, N. Enthalpy-entropy compensation of atomic diffusion originates from softening of low frequency phonons. Nat. Commun. 2020, 11, 3977.

(8) Ceratti, D. R.; Rakita, Y.; Cremonesi, L.; Tenne, R.; Kalchenko, V.; Elbaum, M.; Oron, D.; Potenza, M. A. C.; Hodes, G.; Cahen, D. Self-healing inside $\mathrm{APbBr}_{3}$ halide perovskite crystals. Adv. Mater. 2018, 30, 1706273.

(9) Nie, W.; Blancon, J.-C.; Neukirch, A. J.; Appavoo, K.; Tsai, H.; Chhowalla, M.; Alam, M. A.; Sfeir, M. Y.; Katan, C.; Even, J.; Tretiak, S.; Crochet, J. J.; Gupta, G.; Mohite, A. D. Light-activated photocurrent degradation and self-healing in perovskite solar cells. Nat. Commun. 2016, 7, 11574.

(10) Rakita, Y.; Lubomirsky, I.; Cahen, D. When defects become 'dynamic': halide perovskites: a new window on materials? Mater. Horiz. 2019, 6, 1297-1305.

(11) Almora, O.; García-Batlle, M.; Garcia-Belmonte, G. Utilization of temperature-sweeping capacitive techniques to evaluate band gap defect densities in photovoltaic perovskites. J. Phys. Chem. Lett. 2019, 10, 3661-3669.

(12) Futscher, M. H.; Gangishetty, M. K.; Congreve, D. N.; Ehrler, B. Quantifying mobile ions in perovskite-based devices with temperature-dependent capacitance measurements: frequency versus time domain. J. Chem. Phys. 2020, 152, 044202.

(13) Shockley, W.; Read, W. T. Statistics of the recombinations of holes and electrons. Phys. Rev. 1952, 87, 835-842.

(14) Hall, R. N. Electron-hole recombination in germanium. Phys. Rev. 1952, 87, 387.

(15) Heiser, T.; Weber, E. Transient ion-drift-induced capacitance signals in semiconductors. Phys. Rev. B: Condens. Matter Mater. Phys. 1998, 58, 3893-3903. 
(16) Heiser, T.; Mesli, A. Determination of the copper diffusion coefficient in silicon from transient ion-drift. Appl. Phys. A: Solids Surf. 1993, 57, 325-328.

(17) Lazarus, D. Diffusion studies of vacancies and impurities. J. Phys. Radium 1962, 23, 772-778.

(18) Walter, T.; Herberholz, R.; Müller, C.; Schock, H. W. Determination of defect distributions from admittance measurements and application to $\mathrm{Cu}(\mathrm{In}, \mathrm{Ga}) \mathrm{Se}_{2}$ based heterojunctions. J. Appl. Phys. 1996, 80, 4411-4420.

(19) Lang, D. V. Deep-level transient spectroscopy: A new method to characterize traps in semiconductors. J. Appl. Phys. 1974, 45, 3023-3032.

(20) Maier, J. Electrochemical investigation methods of ionic transport properties in solids. Solid State Phenom. 1994, 39-40, $35-60$.

(21) Riess, I. Mixed ionic-electronic conductors-material properties and applications. Solid State Ionics 2003, 157, 1-17.

(22) Pockett, A.; Eperon, G. E.; Sakai, N.; Snaith, H. J.; Peter, L. M.; Cameron, P. J. Microseconds, milliseconds and seconds: deconvoluting the dynamic behaviour of planar perovskite solar cells. Phys. Chem. Chem. Phys. 2017, 19, 5959-5970.

(23) Jacobs, D. A.; Shen, H.; Pfeffer, F.; Peng, J.; White, T. P.; Beck, F. J.; Catchpole, K. R. The two faces of capacitance: New interpretations for electrical impedance measurements of perovskite solar cells and their relation to hysteresis. J. Appl. Phys. 2018, 124, 225702.

(24) Moia, D.; Gelmetti, I.; Calado, P.; Fisher, W.; Stringer, M.; Game, O.; Hu, Y.; Docampo, P.; Lidzey, D.; Palomares, E.; Nelson, J.; Barnes, P. R. F. Ionic-to-electronic current amplification in hybrid perovskite solar cells: ionically gated transistor-interface circuit model explains hysteresis and impedance of mixed conducting devices. Energy Environ. Sci. 2019, 12, 1296-1308.

(25) Lyubomirsky, I.; Rabinal, M. K.; Cahen, D. Room-temperature detection of mobile impurities in compound semiconductors by transient ion drift. J. Appl. Phys. 1997, 81, 6684-6691.

(26) Futscher, M. H.; Lee, J. M.; McGovern, L.; Muscarella, L. A.; Wang, T.; Haider, M. I.; Fakharuddin, A.; Schmidt-Mende, L.; Ehrler, B. Quantification of ion migration in $\mathrm{CH}_{3} \mathrm{NH}_{3} \mathrm{PbI}_{3}$ perovskite solar cells by transient capacitance measurements. Mater. Horiz. 2019, 6, 1497-1503.

(27) Reichert, S.; Flemming, J.; An, Q.; Vaynzof, Y.; Pietschmann, J.F.; Deibel, C. Ionic-defect distribution revealed by improved evaluation of deep-level transient spectroscopy on perovskite solar cells. Phys. Rev. Appl. 2020, 13, 034018.

(28) Samiee, M.; Konduri, S.; Ganapathy, B.; Kottokkaran, R.; Abbas, H. A.; Kitahara, A.; Joshi, P.; Zhang, L.; Noack, M.; Dalal, V. Defect density and dielectric constant in perovskite solar cells. Appl. Phys. Lett. 2014, 105, 153502.

(29) Yang, W. S.; Park, B.-W.; Jung, E. H.; Jeon, N. J.; Kim, Y. C.; Lee, D. U.; Shin, S. S.; Seo, J.; Kim, E. K.; Noh, J. H.; Seok, S. I. Iodide management in formamidinium-lead-halide-based perovskite layers for efficient solar cells. Science 2017, 356, 1376-1379.

(30) Rosenberg, J. W.; Legodi, M. J.; Rakita, Y.; Cahen, D.; Diale, M. Laplace current deep level transient spectroscopy measurements of defect states in methylammonium lead bromide single crystals. J. Appl. Phys. 2017, 122, 145701.

(31) Xu, W.; et al. Rational molecular passivation for highperformance perovskite light-emitting diodes. Nat. Photonics 2019, 13, 418-424.

(32) Reichert, S.; Goetz, K.; Wöpke, C.; Vaynzof, Y.; Deibel, C. Open-circuit voltage limitation by surface recombination in perovskite solar cells arXiv Preprint, Dec 16, 2020, arXiv:2012.08953 [condmat.mtrl-sci].

(33) Li, H.; Shi, J.; Deng, J.; Chen, Z.; Li, Y.; Zhao, W.; Wu, J.; Wu, H.; Luo, Y.; Li, D.; Meng, Q. Intermolecular $\pi-\pi$ conjugation selfassembly to stabilize surface passivation of highly efficient perovskite solar cells. Adv. Mater. 2020, 32, 1907396.

(34) Zheng, X.; Chen, B.; Dai, J.; Fang, Y.; Bai, Y.; Lin, Y.; Wei, H.; Zeng, X. C.; Huang, J. Defect passivation in hybrid perovskite solar cells using quaternary ammonium halide anions and cations. Nature Energy 2017, 2, 17102.

(35) Xie, H.; et al. Decoupling the effects of defects on efficiency and stability through phosphonates in stable halide perovskite solar cells. Joule 2021, 5, 1246-1266.

(36) Heo, S.; et al. Deep level trapped defect analysis in $\mathrm{CH}_{3} \mathrm{NH}_{3} \mathrm{PbI}_{3}$ perovskite solar cells by deep level transient spectroscopy. Energy Environ. Sci. 2017, 10, 1128-1133.

(37) Pecunia, V.; Zhao, J.; Kim, C.; Tuttle, B. R.; Mei, J.; Li, F.; Peng, Y.; Huq, T. N.; Hoye, R. L. Z.; Kelly, N. D.; Dutton, S. E.; Xia, K.; MacManus-Driscoll, J. L.; Sirringhaus, H. Assessing the impact of defects on lead-free perovskite-inspired photovoltaics via photoinduced current transient spectroscopy. Adv. Energy Mater. 2021, 11, 2003968.

(38) Rau, U.; Braunger, D.; Herberholz, R.; Schock, H. W.; Guillemoles, J.-F.; Kronik, L.; Cahen, D. Oxygenation and airannealing effects on the electronic properties of $\mathrm{Cu}(\mathrm{In}, \mathrm{Ga}) \mathrm{Se}_{2}$ films and devices. J. Appl. Phys. 1999, 86, 497-505.

(39) Gartsman, K.; Chernyak, L.; Lyahovitskaya, V.; Cahen, D.; Didik, V.; Kozlovsky, V.; Malkovich, R.; Skoryatina, E.; Usacheva, V. Direct evidence for diffusion and electromigration of $\mathrm{Cu}$ in $\mathrm{CuInSe}_{2}$. J. Appl. Phys. 1997, 82, 4282-4285.

(40) Herberholz, R.; Igalson, M.; Schock, H. W. Distinction between bulk and interface states in $\mathrm{CuInSe}_{2} / \mathrm{CdS} / \mathrm{ZnO}$ by space charge spectroscopy. J. Appl. Phys. 1998, 83, 318-325.

(41) Deibel, C.; Dyakonov, V.; Parisi, J. Band bending independent of surface passivation in $\mathrm{ZnO} / \mathrm{CdS} / \mathrm{Cu}(\mathrm{In}, \mathrm{Ga})(\mathrm{S}, \mathrm{Se})_{2}$ heterojunctions and $\mathrm{Cr} / \mathrm{Cu}(\mathrm{In}, \mathrm{Ga})(\mathrm{S}, \mathrm{Se})_{2}$ Schottky contacts. Appl. Phys. Lett. 2003, $82,3559-3561$

(42) Rau, U.; Schock, H. Electronic properties of $\mathrm{Cu}(\mathrm{In}, \mathrm{Ga}) \mathrm{Se}_{2}$ heterojunction solar cells-recent achievements, current understanding, and future challenges. Appl. Phys. A: Mater. Sci. Process. 1999, 69, $131-147$.

(43) Guillemoles, J.-F.; Rau, U.; Kronik, L.; Schock, H.-W.; Cahen, D. $\mathrm{Cu}(\mathrm{In}, \mathrm{Ga}) \mathrm{Se}_{2}$ solar cells: Device stability based on chemical flexibility. Adv. Mater. 1999, 11, 957-961.

(44) Deibel, C.; Dyakonov, V.; Parisi, J.; Palm, J.; Zweigart, S.; Karg, F. Influence of damp heat testing on the electrical characteristics of $\mathrm{Cu}(\mathrm{In}, \mathrm{Ga})(\mathrm{S}, \mathrm{Se})_{2}$ solar cells. Thin Solid Films 2002, 403-404, 325330.

(45) Herberholz, R.; Walter, T.; Müller, C.; Friedlmeier, T.; Schock, H. W.; Saad, M.; Lux-Steiner, M. C.; Alberts, V. Meyer-Neldel behavior of deep level parameters in heterojunctions to $\mathrm{Cu}(\mathrm{In}, \mathrm{Ga})$ (S,Se) ${ }_{2}$. Appl. Phys. Lett. 1996, 69, 2888-2890.

(46) Weiss, T. P.; Nishiwaki, S.; Bissig, B.; Carron, R.; Avancini, E.; Löckinger, J.; Buecheler, S.; Tiwari, A. N. Injection current barrier formation for $\mathrm{RbF}$ postdeposition-treated $\mathrm{Cu}(\mathrm{In}, \mathrm{Ga}) \mathrm{Se}_{2}$-based solar cells. Adv. Mater. Interfaces 2018, 5, 1701007.

(47) Werner, F.; Wolter, M. H.; Siebentritt, S.; Sozzi, G.; Di Napoli, S.; Menozzi, R.; Jackson, P.; Witte, W.; Carron, R.; Avancini, E.; Weiss, T. P.; Buecheler, S. Alkali treatments of $\mathrm{Cu}(\mathrm{In}, \mathrm{Ga}) \mathrm{Se}_{2}$ thin-film absorbers and their impact on transport barriers. Prog. Photovoltaics 2018, 26, 911-923.

\section{NOTE ADDED AFTER ASAP PUBLICATION}

This paper was published ASAP on December 7, 2021 with an error in Figure 4. The figure was corrected, and the revised paper was reposted on December 9, 2021. 